\title{
Knowledge and networks: An experimental test of how network knowledge affects coordination
}

\author{
Daniel Enemark ${ }^{\mathrm{a}}$, Mathew D. McCubbins ${ }^{\mathrm{b}}$, Nicholas Weller ${ }^{\mathrm{c}, *}$ \\ a University of Southern California, United States \\ ${ }^{\mathrm{b}}$ Marshall School of Business, Gould School of Law, Department of Political Science, University of Southern California, United States \\ ${ }^{\mathrm{c}}$ Department of Political Science, School of International Relations, University of Southern California, United States
}

\section{A R T I C L E I N F O}

\section{Keywords:}

Information

Experimental methods

Coordination

Communication/information networks

\begin{abstract}
A B S T R A C T
Scholars in the social sciences use network theory to study a range of collective action problems. Often the goal is to identify how the structure of the network affects efforts to coordinate or cooperate, and research suggests that adding connections to a network can improve the performance of groups faced with such tasks. On the other hand, theory and empirics also suggest that additional connections can degrade the performance of a network. If connections can have negative effects then it is important to consider if there are alternatives to adding connections to a network that can also improve network performance. Because a primary function of connections in a network is to disseminate information, providing individuals with more information about the network may act as a substitute for adding connections to a network. We test experimentally whether providing subjects with more information about the structure of networks can improve coordination. We find that a more complete view of the network leads to faster coordination, but the magnitude of this effect depends on network structure. These results suggest that changing what actors know about a network can improve outcomes without having to add connections that may impede overall performance.
\end{abstract}

(c) 2012 Elsevier B.V. All rights reserved.

\section{Introduction}

Large-scale coordination is a central challenge in politics. Examples include choosing political leaders (Calvert, 1992), organizing collective action (Chong, 1991; Weingast, 1997), developing international technological standards (Morrow, 1994), and abandoning harmful cultural practices (Mackie, 1996). As Niou and Ordeshook (1994) put it, the challenge of coordination is "omnipresent," playing a role in literally every major social process. Recently, social scientists have begun to study how the shape of social and institutional networks affects our ability to solve coordination problems such as political participation and protests (McClurg, 2003; Chwe, 2000), natural resource management (Scholz et al., 2008), international trade and diplomacy (Hafner-Burton and Montgomery, 2008), and public goods provision (Bramoullé and Kranton, 2007).

Some scholars have argued that either adding connections to a network or changing the structure of the existing connections can promote successful coordination (Gould, 1993; Cassar, 2007; Watts and Strogatz, 1998) but changing the structure of connections can create new dependencies and therefore more obstacles to collective performance (Chwe, 2000; Enemark et al., 2011; Siegel, 2009; Lazer

\footnotetext{
* Corresponding author. Tel.: +1 2317401668.

E-mail address: nweller@usc.edu (N. Weller).
}

and Friedman, 2007; Jackson et al., 2012). Watts (2003) considered identifying the likely effects of adding connections or changing network structure a particularly vexing problem. Given that changes in network structure can have negative effects, it behooves us to search for an alternative to adding connections when faced with networked coordination problems.

This paper investigates a method for facilitating networked coordination that may pose less risk than modifying the structure of the network-namely increasing actors' view of the network (or in other words, to invest technologies that allow individuals to observe those to whom they are not directly connected). In some circumstances, a more complete view of the network may facilitate coordination that takes place over networks. We present experimental evidence that individuals are able to coordinate more quickly over networks when they can observe not only their neighbors, but their neighbors' neighbors or even the full network, but this effect depends on network structure and more information does not always lead to faster coordination.

\section{Networks in Congress and international relations}

Theoretical models and empirical studies of networks have examined how the structure of a network affects outcomes for a variety of tasks. Examples of scholars using network theories and analogies are too numerous to review in any detail, but to give a 
sense of how common and diverse the applications are, we discuss two prominent examples-networks in Congress and networks in international relations.

\subsection{Congressional networks}

Scholars studying Congress have used a network approach to study the organization of congressional committees and delegation both to committees and bureaucratic agents. The general idea in this literature is that congressional actors intentionally design networks to facilitate their political goals. For instance, Aberbach (1990) studies the information networks that congressional committees use to monitor the actions of various agencies. Although there is heterogeneity in the types and qualities of network developed by different congressional committees, Aberbach claims that overall the "well-developed information networks" aid Congress's attempts to learn about the various agents under their control. One of the most well-developed mechanisms for learning about others is via a network of fire alarms (McCubbins and Schwartz, 1984) that Congress intentionally designs and modifies depending on its political goals and constraints.

Congress also develops networks that are used for internal purposes. For instance, Bradbury et al. (2008) describe how whip organizations serve as networks for transmitting information that helps congressional leaders draft and pass legislation. Overall, then it is clear that scholars have used network analogies to help understand the various relations between members of congress, committees, agencies and interest groups. In the congressional setting scholars typically assume that adding links to the network will make the network more functional.

\subsection{International trade and diplomacy networks}

Network theory and analysis have also been applied to the study of global settings-often looking at networks created by the interaction of countries with each other (see Kahler, 2009; HafnerBurton et al., 2009 for recent reviews). Scholars of international relations have long argued that countries are not independent of each other and that networks provide a way to model and study the dependencies between countries. Bradley and Kelly (2008) write: "Most nations today participate in a dense network of international cooperation that requires them to grant authority to international actors." Kahler (2009) provides an overview of different applications of network studies in international relations. In general, scholars have used the network approach to capture the relational nature of international politics and the way that structure and agency interact to produce outcomes.

Scholars studying the diffusion of public policy (Simmons and Elkins, 2005; Gleditsch and Ward, 2006, 2008) theorize about and model the effect of connections between countries on the likelihood that one country's policy choices affect another's. In this same vein, Cowhey and Mueller (2009) writes, "scholars have used the concept of networks to examine how informal systems of information exchange and coordination can organize actors globally." Network models allow scholars to relax the assumption that policy choices are independent across jurisdictions and account for the ways that ties between countries (and the topology of these ties) affect the choice of public policy.

Others studying networks in international relations have examined how the ties between countries either engender or mitigate conflict. Scholars using this approach make direct use of various concepts from network theory and connect traditional concepts in international relations to measures derived from network theory (Hafner-Burton and Montgomery, 2006; Maoz, 2006; Maoz et al., 2007). Scholars studying conflict have argued that more connections between countries can either prevent or encourage conflict.
The idea that connections could lead to inferior outcomes is crucial and it suggests that if we can control the structure of the network we will want to understand if an additional connection will help or hurt performance.

Of course, network theory has been used to model relational settings in other areas of political science (for a review, see Ward et al., 2011). We focus on these two subtopics simply to provide a sense of how widespread are the uses of network models and analogies in political science. In addition, in both of these settings the networks are largely designed by political actors to achieve goals. For instance, in the examples of congressional committees and delegation, the networks are designed by congressional actors and agencies to help achieve political goals such as information gathering and oversight. Likewise, in the international relations examples the networks are created by intentional interaction between countries and international institutions. In both cases, political actors make choices about network design that influence how effective the network is at helping them achieve some goal. The widespread use of network models in political science should make it clear that understanding how networks affect political outcomes is a central concern to scholars.

\section{The network design problem}

In studies of networks one of the key goals is to identify how the structure of a network affects outcomes. In many political settings (such as the ones we discussed in the prior section) the network structure does not arise randomly, but rather is at least in part a result of conscious design. Scholars have tended to argue that connecting previously unconnected actors is a good idea when designing or modifying a network, and experimental and observational research has implied that adding connections to a network can improve coordination (Kearns et al., 2006; McCubbins et al., 2009; Gould, 1993; Grannovetter, 1974). In particular, network structure can facilitate coordination even when subjects face considerable asymmetry in their payoffs such as in a Battle of the Sexes (McCubbins et al., 2009). The idea that more connections in a network are good underlies the business of social media companies such as Facebook, LinkedIn, MySpace, and Google+, which seek to make it easier for people to connect with each other and thereby share information.

Adding links to a network, however, may not improve performance. Scholars noted years ago in studying road networks that adding a new road to the existing network of roads could actually degrade overall performance (Braess, 1969). This is not just a theoretical curiosity, either. In 1990, New York City closed 42nd street in preparation for Earth Day festivities and many expected traffic to get much worse (Kolata, 1990). However, traffic actually improved with the removal of the street from the traffic network. Recently, computer scientists have turned to studying the phenomenon called Braess's Paradox across a host of network structures and they find that it is likely to occur quite commonly (Chung and Young, 2010).

Political scientists have also noted that adding connections to a network may either improve or worsen outcomes:

"connectedness may impose constraints on autonomy as well as offer opportunities for influence.... States that are part of an alliance network may find themselves in conflicts they would rather avoid; trade ties can be used for economic sanctions; normative bonds are deployed to force compliance through naming and shaming; and telephone and email records can be used to destroy a terrorist network." Hafner-Burton et al. (2009)

The possibility that a new connection could impair activity in a network is also identified by Fowler (2005) and Siegel (2009) in 
studying how social networks can affect behavior such as voter turnout. Watts (2003) noted the general problem of how to add connections to a network "If adding links at random isn't a good way to reduce information congestion, what is? In general, this is a hard question to answer, requiring as it does a balance between local capacity constraints and global (system-wide) performance."

The negative effect of an additional connection being added to a network has also been demonstrated experimentally, Enemark et al. (2011) show that in a distributed coordination task the addition of connections that change the solution space to a coordination problem can significantly impair a group's ability to find a solution. Previous research has identified the negative effects of connections in both coordination and cooperation tasks, so the findings cover a large amount of commonly studied social science settings. All of these results should caution us against assuming that adding connections to a network will improve the overall performance of the actors in that network.

One alternative to adding new connections may be to give actors a more complete view of the network. For instance, if actors can see both their immediate neighbors and their neighbors' neighbors, this may improve their ability to coordinate or cooperate. After all, both more connections and a more complete network view give actors more information about the choices of others. When network connections represent real connections-and therefore dependencies-between actors, the advantage of the more complete view is that it avoids the possibility that an added connection will negatively affect overall performance. In this paper we design a variety of experiments to test whether a more complete view of the network can act as a substitute for more connections in a network. We focus on coordination problems in our experiments because they are a widely studied and important aspect of social situations. We briefly discuss the ubiquity of coordination in the following section.

\section{Experimental tests of the effect of network knowledge on coordination}

To experimentally test how the design of a network affects the performance of the network we must select both a range of networks and a class of tasks for subjects to complete. We, therefore, combine a coordination task with various networks. Easley and Kleinberg (2010) note that these tools are essential to understanding behavior:

We begin with the two main bodies of theory that we will be building on-graph theory and game theory. These are theories of structure and behavior respectively: Graph theory is the study of network structure, while game theory provides models of individual behavior in settings where outcomes depend on the behavior of others.

Our experiment involves 16-node networks in which each subject controls a node and the task is to solve a decentralized coordination problem. We study two forms of coordination: consensus and anti-coordination. In the consensus form the task is for all players to choose the same color and in the anti-coordination form the task is for players to choose a different color than the other nodes to which they are connected. In both forms of the task the players have two colors to choose from.

In studying the effects of network structure on distributed coordination, we face two formidable obstacles to generalizability: the staggering topological diversity of networks, and the wide range of coordination games that humans play. The number of distinct network structures possible with just 16 nodes is $6.4 \times 10^{22}$. To complicate matters, human networks-both organic and designed-often exhibit peculiar properties such as a scale-free degree distribution (see, for example, Ebel et al., 2002), and at large scales may have partitioning characteristics that do not match any of the common models for random network generation (Leskovec et al., 2009). Thus there are no clear criteria for generating the most representative or relevant sample of networks from the vast variety of possible networks. Moreover, the degree to which a particular network topology helps or hinders coordination depends upon the nature of the task itself. For instance, a network that fosters efficient consensus may impede both anti-coordination tasks like fashion or marketplace competition and matching games like the marriage or labor market.

Because the space of networked coordination tasks is essentially infinite, we must be humble in our claims of generalizability, and acknowledge that no matter how many experimental tasks and networks and trials we run, there is no way to demonstrate that the effects we observe are universal. We do, however, attempt to offer as broad a test as practicable. We use two different forms of coordination and multiple network structures because the replication of results across a range of environments is one method to improve the generalizability of our findings (Shadish et al., 2001).

In both tasks subjects all share a common incentive to solve the problem and there is no conflict in preferences over the various coordinated outcomes. Coordination is a widely relevant problem to study because a great many political, economic and social situations involve coordination (i.e. multiple equilibria are present), and therefore our findings are about a class of problems that are important across many environments. Pure coordination games are ideal for studying the effect of information because information is a key factor that determines if players can identify and agree upon one of the multiple solutions to the coordination problem (Schelling, 1960). In addition, coordination problems involve considerable interdependency between actors, and networks can model both the information structure and the interdependencies that determine successful coordination.

In the experiment, each subject controls one node in the network so that coordination is the result of distributed actions. ${ }^{1}$ If the subjects reach a successful group solution to the coordination problem, then each subject earns $\$ 1$ and if they fail to reach a solution they earn nothing. Subjects are all read a set of instructions together to ensure common knowledge of the rules of the game and then quizzed on the instructions. Subjects play the game anonymously, hidden behind partitions in a classroom. Network structure varies from trial to trial (it is randomly assigned across trials, as a withinsubjects design) and subjects are assigned to a node randomly at the beginning of each trial within a given experiment. In addition, the two available colors (actions) are randomized across subjects and trials (meaning that color 1 may appear as blue for one subject and pink for another). These measures prevent learning over the course of the experiment and impede subjects from developing norms about how to use colors to coordinate. The computer monitor displays the following information during each experimental session.

- Information view: we modify what subjects know about the network and what they know about the nodes to which they are connected. There are four possible information conditions, from the least to the most complete view of the network.

\footnotetext{
${ }^{1}$ In the anti-coordination task, all nodes must choose a different color than that of their neighboring nodes to reach a successful solution. Graph theorists will recognize this as a distributed graph-coloring problem. In the coordination task, all nodes must choose the same color as their neighboring nodes, which in a connected graph, means that all nodes must settle on the same color. In a network that only requires two colors for a successful solution (such as the ones we study) these two tasks are formally equivalent.
} 


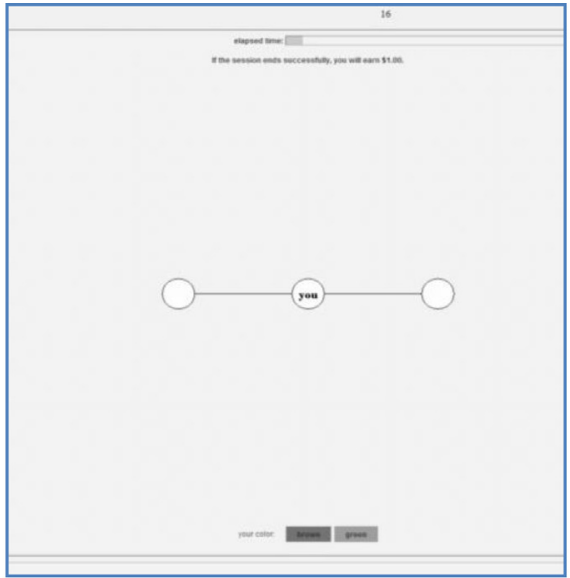

Neighbor View

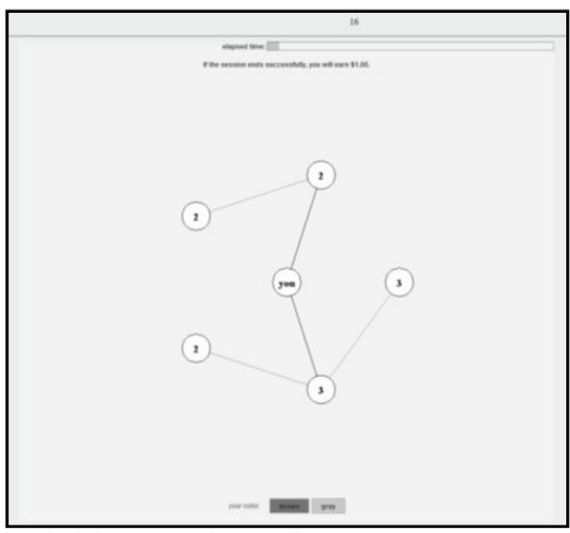

Neighbor + Alter View

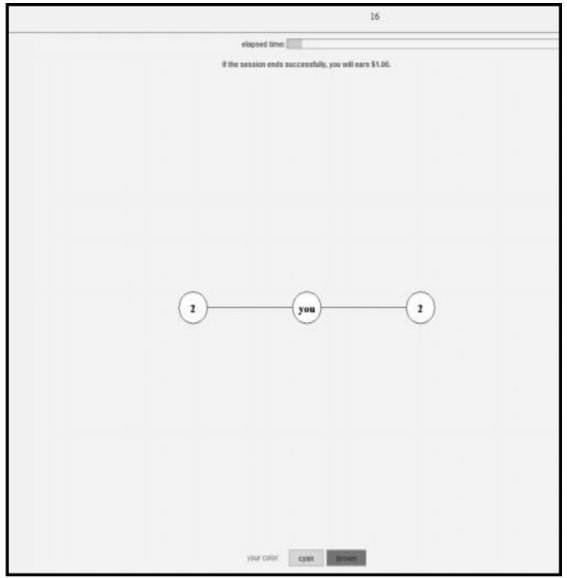

Neighbor + Degree View

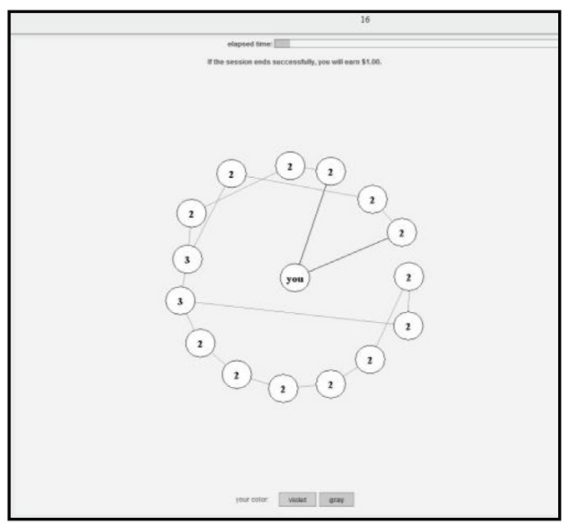

Full Information View

Fig. 1. Experimental interface with each information view.

- Neighbor: subjects see the nodes to which they are connected and the color of each node as they change throughout the experiment.

- Neighbor + Degree: subjects see the nodes to which they are connected and the number of connections (degree centrality) of each of those nodes.

- Neighbor + Alters: subjects see everything in the Degree view, plus they see their neighbors' neighbors.

- Full Network: subjects see a representation of the entire network. ${ }^{2}$

- Choice of actions: subjects can see the two alternative actions available to them, presented to subjects as colors they can choose for their node.

- Time remaining: subjects can see how much time is remaining in the trial.

In Fig. 1 we present a sample screen shot from each of the four different information views. Depending on the information view, subjects' knowledge of the network varies. The key feature of this experimental design is that we can modify the information

\footnotetext{
2 Even though they see the full network, this is not equivalent to seeing simply a tally of the number of people who have picked each color, because the coordination problem requires being a different color than those to whom one is connected, so the structure of the network matters in addition to the number of nodes of each color If it were a standard consensus problem the network structure would be irrelevant in the full information view, as the only thing in a "full" view that matters is the number of nodes that are each color.
}

environment of individuals (what they know about the network) and the network structure independently.

\subsection{Network selection for experiments}

The 16-node networks we use in our experiments are clearly much smaller than many of the social networks studied in observational research. However, even with only 16 nodes the number of possible networks we could design is very large. We have to choose from this universe a set of networks to study. Accordingly, we selected networks that varied in both the both number of connections and the distribution of those connections.

In studying anti-coordination we must select networks for comparison that allow the game to be solved with the same number of colors (in these experiments that is two colors). For instance, if we add any triangles to a network the network then requires three colors to solve an anti-coordination task, and cannot be compared to a network that requires only two colors. Therefore, we select the networks in Fig. 3. In selecting these networks we focused mostly on identifying networks with a varying number of connections because prior work has shown that connections can facilitate coordination by spreading information among the actors in the network (Kearns et al., 2006; McCubbins et al., 2009; Enemark et al., 2011) (Fig. 2).

In our experiments on consensus we chose the five different networks displayed in Fig. 3. We chose to use different networks in the consensus tasks than we used for the anti-coordination task, in an effort to maximize the diversity of environments in 


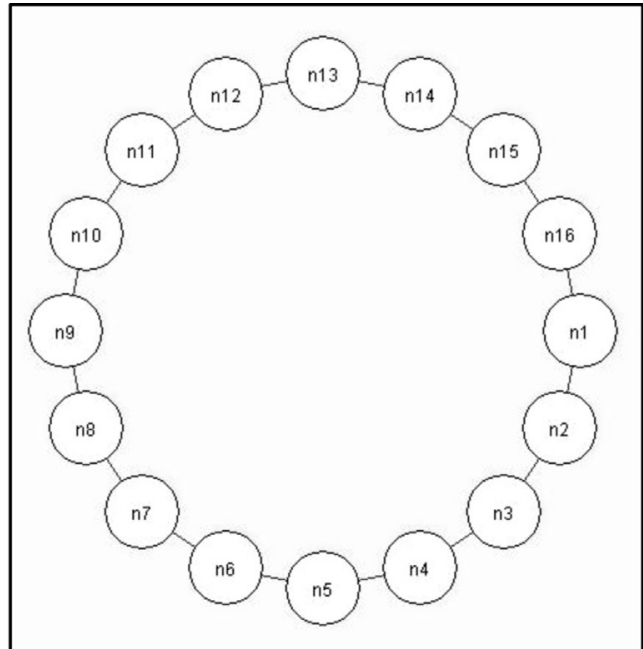

Cycle

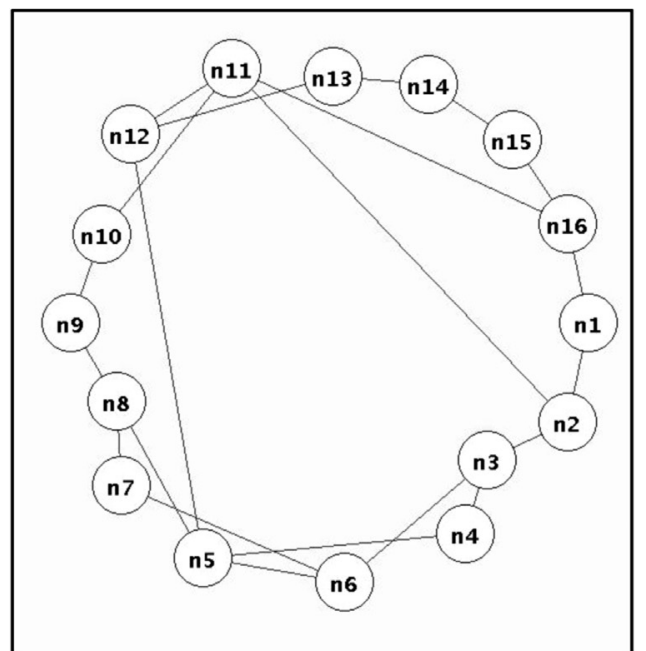

Cycle Plus

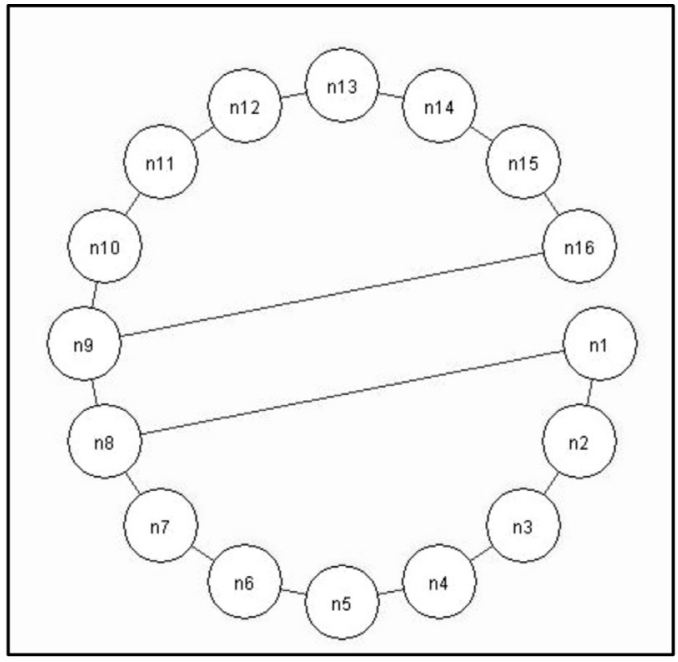

Barbell

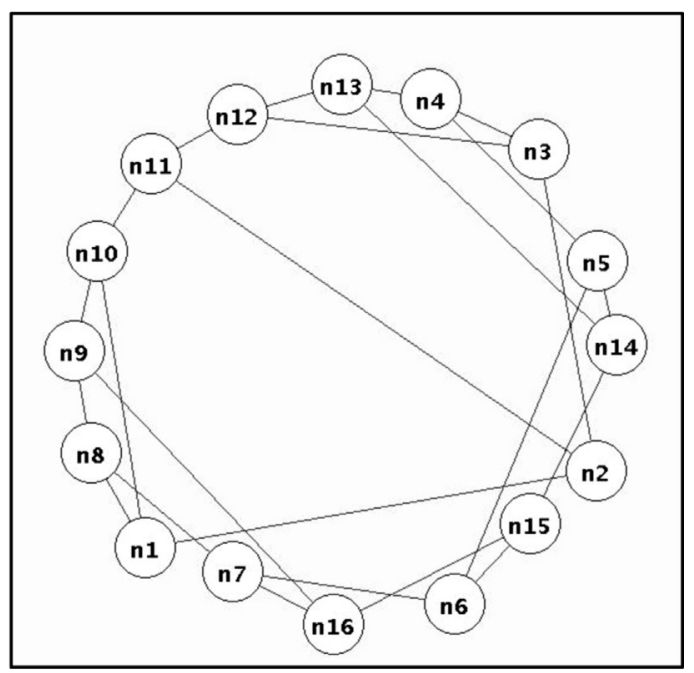

Cylinder

Fig. 2. Networks used in anti-coordination experiments.

which to observe the effect of information on coordination. The use of different networks for the two tasks was prudent because our investigation is focused on the effect of an expanded network view, and not on a comparison of behavior in consensus and anti-coordination tasks. If changing the information views affects coordination across both different tasks and different networks, then can be more confident to have identified an effect that does not depend on the precise networks or coordination tasks. Also, in a consensus task additional connections beyond the minimally connected network do not constrain the number of solutions to the task, so we are free to design networks that mirror a range of common human networks without concern for how the network affects the solution space to the problem. This allows for the inclusion of triangles (or triads, as they are called in sociology), which are a crucial component of many human networks (Simmel, 1950). The two networks labeled "binomial" were generated using the Erdős-Rényi model, so that each of the 120 possible connections of the 16 -node network exists with independent probability $p$, where $p$ is one-fifth

Table 1

Statistics for networks used in experiment.

\begin{tabular}{|c|c|c|c|c|}
\hline & Network & Average degree & Variance in betweenness centrality & Variance in degree \\
\hline \multirow[t]{4}{*}{ Anti-coordination networks } & Cycle & 2 & 0 & 0 \\
\hline & Barbell & 2.13 & 294.40 & 0.12 \\
\hline & Cycle plus & 2.625 & 101 & 1 \\
\hline & Cylinder & 3 & 33 & 0 \\
\hline \multirow[t]{5}{*}{ Consensus networks } & Binomial20 & 3.38 & 95.23 & 1.58 \\
\hline & Stars & 5.25 & 109.85 & 23.4 \\
\hline & Mixture & 7 & 0 & 0 \\
\hline & Segregation & 7 & 116.81 & 0 \\
\hline & Binomial50 & 7.88 & 7.26 & 6.65 \\
\hline
\end{tabular}




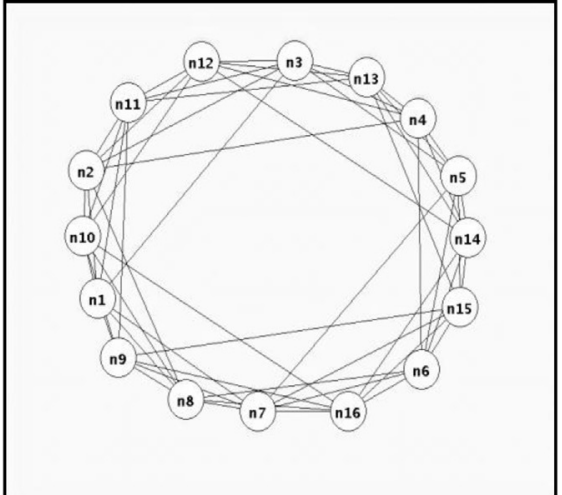

Mixture

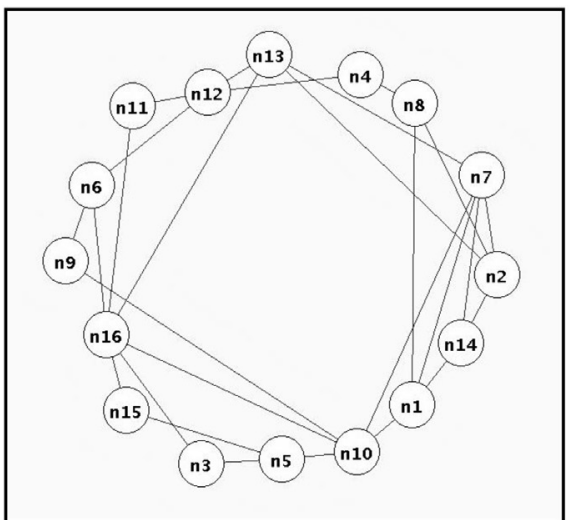

Binomial Random, 20

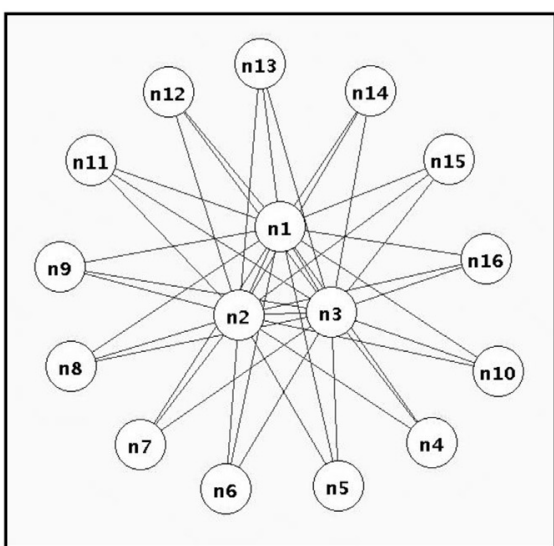

Stars

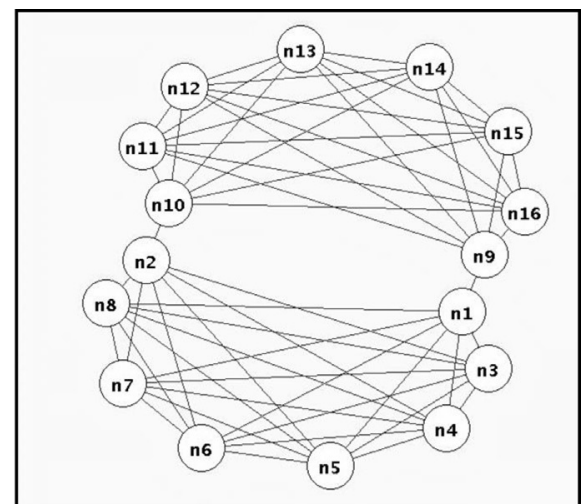

Segregation

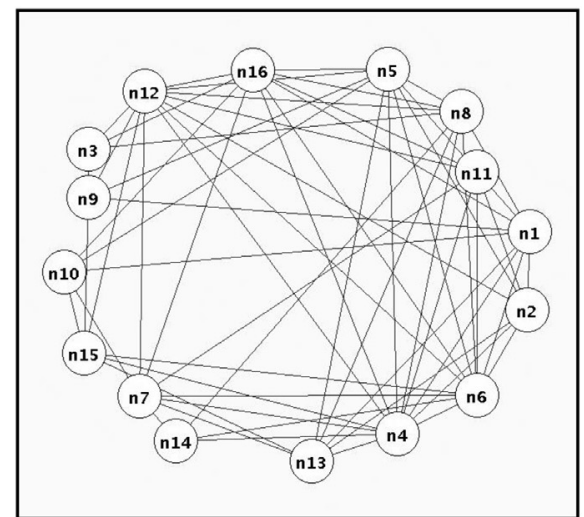

Binomial Random, 50

Fig. 3. Networks used in consensus experiments.

in Binomial20 and one half in Binomial50. The five networks we chose provide a good range of network characteristics as shown in Table 1.

\subsection{Expectations}

The connections in our network define the coordination problem and provide information to subjects about the actions of the subjects to whom they are directly connected in the network. The varying information views display the actions of these immediate neighbors and, in some cases, the actions of and connections between other nodes. In coordination games information is a key determinant of coordination, which leads us to expect that the structure of the network and the information view will interact with each other. Therefore, we expect:

1. A more complete view of the network will lead to faster coordination.

2. The effect of the information view will depend on network structure.

The more complete view of the network provides subjects with both more information about the overall structure of the network as well as information about the choices of other nodes in the network. Both of these attributes should make it easier for subjects to adjust their behavior to what others have done, which 
Table 2

A more complete network view can make anti-coordination easier.

\begin{tabular}{ll}
\hline & DV: time to coordinate \\
\hline Cycle & $70,917(23,429)^{* *}$ \\
Cycle plus & $25,766(21,932)$ \\
Barbell & $13,844(24,054)$ \\
Full network view & $48,034(42,590)$ \\
Full network view in cycle & $-94,173(33,135)^{* *}$ \\
Full network view in cycle plus & $-35,832(32,064)$ \\
Full network view in Barbell & $-15,255(33,987)$ \\
Experiment order & $-1440(1027)$ \\
Constant & $71,739(19,858)^{* *}$ \\
$N$ & 80 \\
Number of groups & 4 \\
\hline
\end{tabular}

Linear multi-level model with a random intercept for each experimental group. Unsuccessful trials are right-censored at $180,000 \mathrm{~ms}$. Excluded network is the cylinder network. Time to coordinate is measured in milliseconds.

*Significant at 0.05 level.

Significant at 0.01 level.

should make coordination easier. It should be noted that changes in the information view modify two attributes of the experimental situation at the same time. This means that we cannot make inferences about how knowledge of structure (absent knowledge of others' choices) affects the outcome. However, we utilized this approach because it seems that in real-world settings providing individuals with knowledge of the network structure would also involve giving them information about others' choices. ${ }^{3}$ For example, improving the monitoring of congressional agencies implies knowledge of both the connections among agencies and firms and also some knowledge of the actions taken by both sets of actors. Further experiments could give subjects a static view of the network without information about others choices to isolate precisely if network knowledge affects behavior even if it does not include knowledge of other's actions (Table 2).

We expect that the effect of a more complete view will depend on the number of connections and variance in degree across nodes in the network. Connections transmit information, so if a network has more connections it already provides subjects with greater information about the actions of others and therefore a more complete view of the network will be less useful. Degree variance can create focal nodes, allowing distributed actors to engage in "leader election." Scholars have argued that leaders can help resolve coordination problems (Wilson and Rhodes, 1997; Calvert, 1992). In the case of both high average degree and high variance in degree, if the network structure makes coordination easier, then the more complete view of the network should have less effect on coordination time.

Our expectations are about the effect of network structure and information environment on group behavior, not individual behavior. Achieving causal identification about how the treatments (network structure and information views) affect individuals in this experiment is problematic because of SUTVA violations (Rubin, 1990) and social threats to internal validity (Shadish et al., 2001). If we change an attribute for a single individual (i.e. a node's degree), then it might affect the behavior of the other nodes in the network. To put it another way there is an interaction between each node's degree and the overall behavior of the network, and therefore we cannot draw reliable inferences about individual choices in this setting.

\footnotetext{
3 An additional practical benefit is that, since we expect knowledge about network structure and knowledge about others' actions to have effects of the same sign, combining these treatments is more likely to produce a measureable effect. Given the expense of conducting experiments in which the unit of analysis is the behavior of 16 individuals, we could not conduct enough trials to measure minor effects.
}

\subsection{The irrelevance of equilibrium strategies}

We have not presented an extensive form of the game and derived equilibrium strategies. Instead, we focus on whether the group, as a whole, can properly color the graph. There are three reasons for this. First, our experiments are designed to study collective outcomes. Our interest is in whether groups can solve coordination problems, so we do not focus on individual's strategies in the game. Second and most importantly, as others have noted in coordination games that are dynamic and feature communication there are typically so many possible equilibrium strategies that it is not useful to focus on them (Choi et al., 2008; Echenique and Yariv, 2011).

Finally, in our network games, the vast majority of Nash equilibria do not represent a solution to the coordination problem. For example, consider a 16-player consensus game in which players can choose red or green. Just to simplify the discussion, imagine that in this game, all players choose a color (red or green) simultaneously. Of course, any combination of choices that yields a solution to the consensus task is an equilibrium, because all players earn the maximum payoff of $\$ 1$. We can call this a "solution equilibrium," and its basis of attraction is that no player would deviate from a solution outcome to achieve a non-solution outcome.

Unfortunately, there is a second class of equilibria that we might call "quagmire equilibria." A quagmire equilibrium is one in which the outcome is negative but more than one player would have to change his action for the group to achieve a positive outcome. Since the requirement for equilibrium is that no single player can unilaterally improve his own payoffs, nearly every possible outcome is in equilibrium. If we consider the pure-strategy Nash equilibria in the simplified consensus game described above, there are two "solution equilibria” and 65,502 “quagmire equilibria." Only 32 outcomes are not pure-strategy Nash equilibria. The coordination games our subjects play become even less tractable to equilibrium analysis when we recognize that play is asynchronous and involves communication and Knightian uncertainty. ${ }^{4}$

\subsection{Why focus on coordination?}

Networks may affect many different outcomes, but in a given study scholars must choose a particular outcome to study when investigating the effects of networks. In this paper we focus on how network structure and actors' information about the network affect a group's ability to solve a coordination problem.

Coordination is clearly an important outcome for social scientists to understand. As Wilson and Rhodes (1997) point out "Coordination problems present a fundamental social puzzle. They are vexing when they occur, but are easily solved with hindsight" (p. 767). In addition, coordination is important in settings as diverse as political protest in the civil rights movement (Chong, 1991); international policy (Niou and Ordeshook, 1994); interaction between nation-states (Snidal, 1985; Stein, 1982); development of shared language (Lewis, 1969); and driving (Lewis, 1969). Niou and Ordeshook (1994) point out that "Because every ongoing social process possesses a multiplicity of equilibria, opportunities to cooperate and the concomitant problem of coordinating to one of

\footnotetext{
${ }^{4}$ In most cases, our subjects do not know (even probabilistcally) the shape of the full network. Because (in anti-coordination tasks) the payoffs depend on the shape of the network, the games our subjects play involve uncertainty as Knight (1921) defined it. The Nash equilibrium solution concept was only recently adapted to the context of simple normal-form games with Knightian uncertainty (Dow and Werlang, 1994), and the authors are not aware of any work defining Nash equilibrium for an extensive-form game with communication and Knightian uncertainty.
} 
these equilibria are omnipresent (p. 210)."5 The ubiquity of coordination makes it an important class of problems to understand.

A very concrete, practical example faces drivers on a daily basis. In everyday life drivers face multiple coordination problems-and the coordination problems involve both consensus and anticoordination. We are all better off if we can agree on a consensus for which side of the road to drive on (Lewis, 1969). Even once that problem is solved (usually by political fiat), drivers on a daily basis face a much more vexing discoordination problem for which centralized solutions do not exist-how to time one's use of the roadway to minimize travel time, subject to a variety of external constraints. The goal in this coordination problem is to NOT drive at the same time as everyone else. Rather, everyone does better if we discoordinate our driving schedules so as not to overuse the roadways.

Suffice it to say, coordination is central to successful political and social outcomes and the institutions that we design can affect the likelihood of coordination. Therefore, understanding what affects coordination is a central concern for social scientists. Given that coordination always involves multiple actors it is also natural to model the network between actors and how this network affects coordination. ${ }^{6}$

\section{Results and analysis}

Using this experimental setup we can test how the information we provided to actors affects their ability to solve a coordination problem. In a pure coordination game without any conflict between individuals the primary obstacle to successful coordination is information about what others are doing. Given this, we expect that a more complete view of the network will facilitate coordination. In addition, prior research (Kearns et al., 2006; McCubbins et al., 2009) demonstrates that increasing the number of network connections can facilitate coordination.

\subsection{Results for anti-coordination}

Our experiments of anti-coordination involve 161 different trials across four different groups of subjects. In 21 of the 161 trials the subjects failed to solve the coordination problem before the 3-min time limit. In Fig. 5 we look at the average time to completion across all our trials for each information view in the anti-coordination experiments. ${ }^{7}$ In general, more information facilitates coordination. The biggest drop in the time to completion occurs when we provide subjects with information about their neighbors' alters, which allows them to understand their neighbors' constraints. $^{8}$ We turn now to a regression model to estimate whether there the effect of the information views differs from network to network. For the sake of presentation we focus on just the neighbor information and full information views. ${ }^{9}$ In addition,

\footnotetext{
${ }^{5}$ Particularly informative discussions of cooperation and coordination occur in Snidal (1985) and Stein (1982). McCubbins et al. (2009) focused directly on situations that involved elements of cooperation and coordination such as the Battle of the Sexes. In this paper we turn to a sole focus on the difficulty of the coordination task.

${ }^{6}$ Other scholars have also found that networks may not affect cooperation (Suri and Watts, 2011), so if we want to study how networks affect behavior it is useful to focus on an outcome likely to be affected by network design.

7 We balance our experiments such that in each information view we have a similar number of trials for each network structure. This helps to ensure that the effect of information view is not due to different networks being used in each condition.

8 These findings are confirmed by a regression in which we estimate the effect of network structure and information view on the time to successful coordination. Code for all analysis, including this robustness check, is available at our data repository: http://dvn.iq.harvard.edu/dvn/dv/NetGames.

9 Again, the model with full interactions between each level of information views and each network is included in the code posted online. The results are consistent
}

we include a dummy variable for each network rather than attempt to measure and include relevant statistics for all the ways our networks differ from each other. This allows us to focus directly on comparisons between networks. This model is also a better match to our experimental design, because the experimental treatments are the networks and information views. ${ }^{10}$

The excluded network is the cylinder, which has the largest number of connections, and we include a dummy variable for the full-information view that is interacted with the three networks-Cycle, CylinderPlus and Barbell. The results demonstrate that the Cycle network is completed significantly slower than the Cylinder, but the other two networks are indistinguishable in our dataset. Consistent with our expectations, the effect of the full information view depends on the network. The full information view only significantly reduces the average time to achieve coordination in the Cycle0 network, which has the fewest number of connections (Fig. 4).

The four networks used for the anti-coordination trials differ in their number of connections and structure, and since connections represent an informational link we expect heterogeneity in the effect of information views such that networks with more connections are less affected by more complete information views. To illustrate this relationship, Fig. 5 displays the time to coordination for the Cycle and the Cylinder network, which are the least- and most-connected of the two networks. ${ }^{11}$ The figure displays time to completion on the $y$-axis and information views (from sparse to complete) along the $x$-axis.

Fig. 5 includes three results worthy of note. First, the two networks differ substantially in their time to completion, particularly in the two lowest information conditions. In both these conditions the Cylinder network is completed much more quickly, and given that connections in these networks do nothing but carry information about others' choices, this result is rather intuitive and matches previous findings showing that, holding constant the difficulty of the underlying task, adding more connections helps groups to solve networked coordination problems (Enemark et al., 2011). Second, as we move rightward on the $x$-axis we see that the effect of increasing actors' information is not constant across the two networks. In the Zero-chord network, which has the fewest connections, there is a considerable decline in the average time to coordinate (an improvement in the group's ability to coordinate). On the other hand, the cylinder network, which has the most connections, does not feature major changes in the time to coordinate as we provide actors with more information about the network. The additional information we provide actors is less valuable if the network itself already provides a great amount of information. Third, by the time we have added the full information view the two networks are nearly identical in their time to completion, removing the relationship between number of connections and coordination. These results indicate that theories about the effect of information must take account of the network structure in which individuals

with the results reported in the paper, but are more difficult to interpret because of the number of iterations.

10 The network statistics in Table 1 also show that moving from one network to another usually modifies multiple attributes of a network (and this is with only three reported attributes). Therefore, controlling for network features via regression adjustments is largely illusory, because we lack overlap on the relevant dimensions between the different networks (King and Zeng, 2006). In addition, if we try to include both a dummy variable for the networks and a measure of the number of connections per network the two are collinear and the regression cannot be estimated.

11 Although not reported for presentation reasons, the Barbell network falls somewhere in between the Cyle and Cylinder network in time to completion and the Cycle Plus network looks more or less like the Cylinder network. Full results are available at: http://dvn.iq.harvard.edu/dvn/dv/NetGames. 


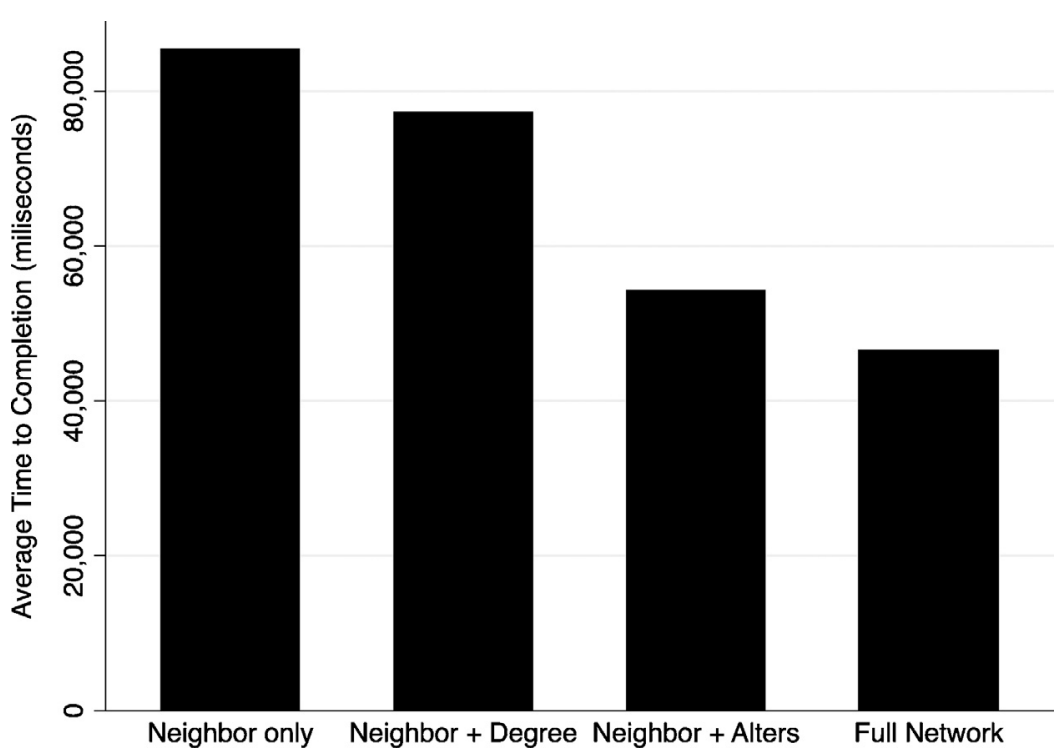

Fig. 4. More information about the network reduces time to solve a coordination problem.

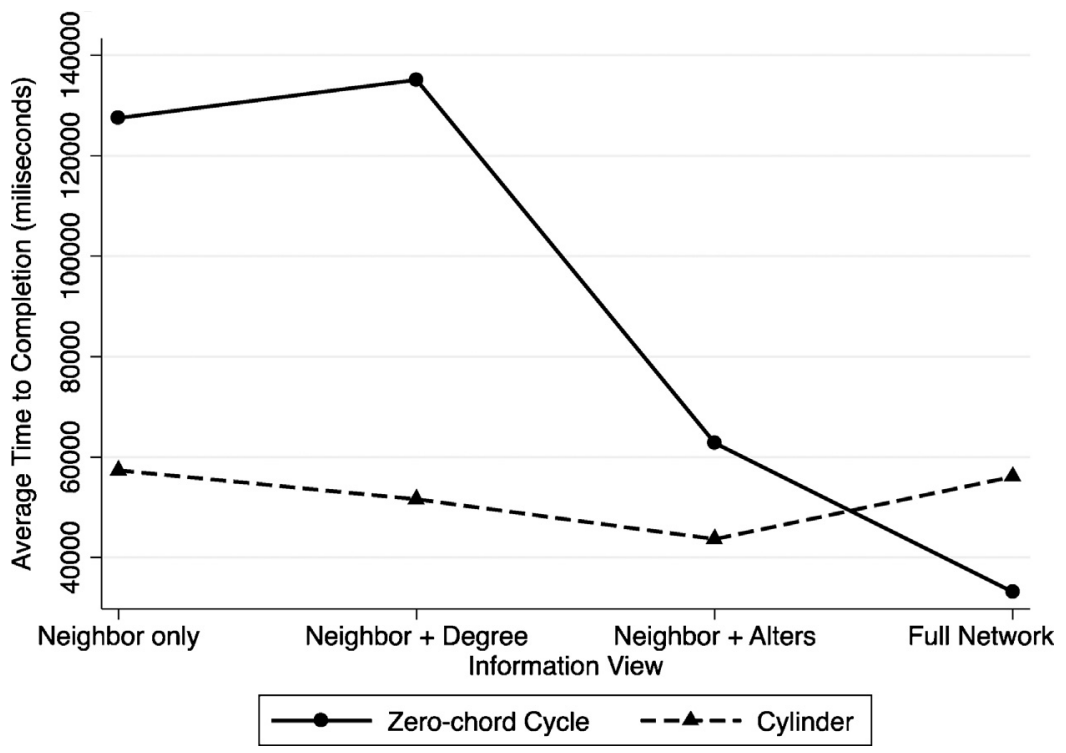

Fig. 5. The effect of deeper information depends on network structure.

act, because the value of a more complete view into the network depends on the network itself.

\subsection{Results for consensus coordination}

To study how consensus is affected by network structure and information views we used the networks displayed in Fig. 3. We conducted experiments with four different groups of undergraduate students who overall completed 166 different experimental trials. All of the trials ended with subjects successfully achieving consensus before the 3-min time limit was reached. The statistics in Table 1 demonstrate that the five networks we use provide cover a wide range of values on three important dimensions of network topology. In these experiments we utilize only the medium and full information views. The difference across all our trials between full information and neighbor + degree information is displayed in Fig. 6, which indicates the main effect that providing subjects with more information about the network facilitates consensus. However, we have reason to believe that this effect will depend on network structure, because of the substitutability between network connections and a more complete information view.

Fig. 7 displays the difference between the two information conditions for the Segregation and Binomial50 networks. In the Binomial50 network, giving subjects a more complete view of the network does not affect the average time to coordinate, but in the Segregation network the more complete information view significantly reduces the time required for groups to coordinate successfully. These two networks are illustrative of the interaction between network structure and information view. ${ }^{12}$ We examine the interaction between network structure and information view statistically in Table 3, where we estimate the effect of network structure and information view using a multi-level linear

\footnotetext{
12 The two networks differ in multiple parameters so it is difficult to attribute the differential effect to any given parameter with much confidence. The results are suggestive that degree variance may be more important than betweenness centrality in facilitating coordination, but without further experiments on different networks that is a hypothesis rather than a result.
} 


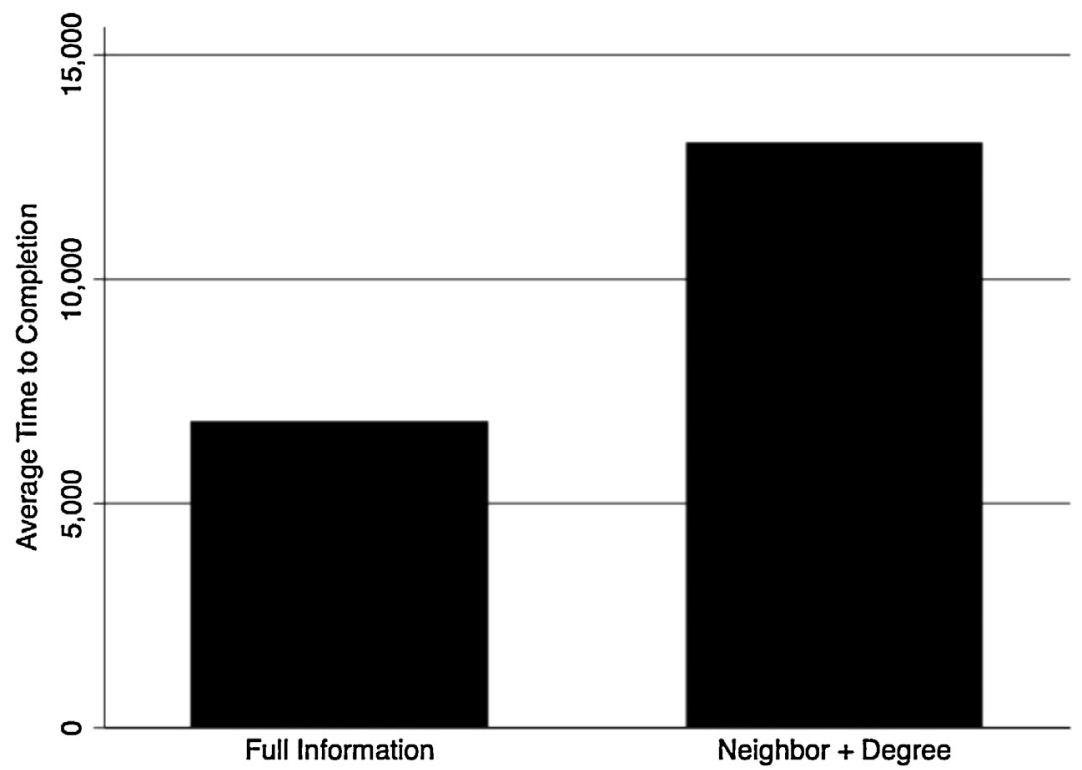

Fig. 6. A deeper information view can facilitate consensus.

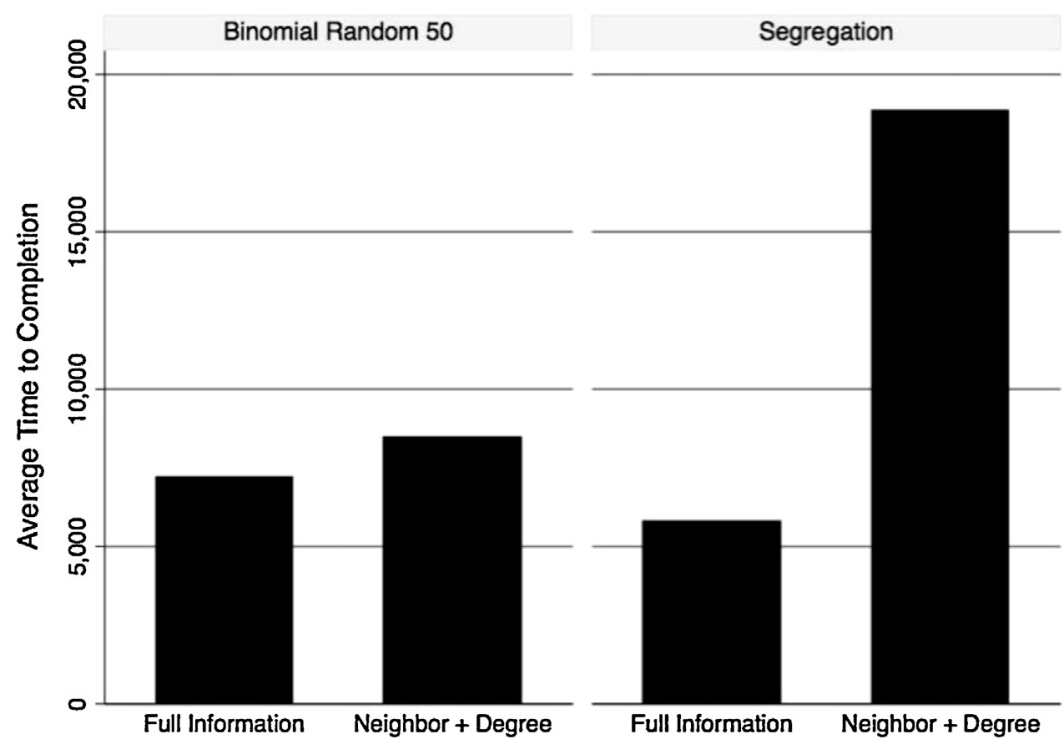

Fig. 7. Effect of deeper information depends on network structure

Table 3

Information can facilitate consensus coordination.

\begin{tabular}{ll}
\hline & DV: time to coordinate \\
\hline Binomial20 & $7946(2560)^{* *}$ \\
Stars & $-1028(3391)$ \\
Mixture & $6617(3386)$ \\
Segregation & $9807(3364)^{* *}$ \\
Full information view & $-1408(2568)$ \\
Full information view in Binomial20 & $-8515(3646)^{*}$ \\
Full information view in stars & $1339(4237)$ \\
Full information view in mixture & $-6972(4274)$ \\
Full information view in segregation & $-10,851(4358)^{* *}$ \\
Experiment order & $40.1(58.9)$ \\
Constant & $7623(2181)^{* *}$ \\
$N$ & 166 \\
Number of groups & 4 \\
\hline
\end{tabular}

Linear multi-level model with a random intercept for each experimental group. Excluded network is the Binomial50 network. Time to coordinate is measured in milliseconds. Unsuccessful trials are right-censored at $180,000 \mathrm{~ms}$.

* Significant at 0.05 level.

** Significant at 0.01 level. regression model. We include a dummy variable for each network for reasons discussed in the previous section, and the excluded network is the Binomial50 network.

The results indicate that the move from medium to full information significantly reduces coordination time in the Binomial20 and segregation networks, but does not have a significant effect in the other networks. This is consistent with the idea that the effect of information will depend on network structure. Indeed the Binomial20 has the fewest number of connections of the networks used in the consensus experiments. The segregation network has considerably more connections than Binomial20, but has no variance in degree and therefore no nodes that may be able to function as leaders to facilitate coordination. However, if degree variance is the obstacle to coordination then we should observe a similar effect in the mixture network, which we do not. The mixture and segregation networks differ substantially in betweenness centrality, and while this may explain the difference, we know little about the effect of betweenness centrality on coordination, so we plan to run further experiments to investigate this relationship. 
The results from the consensus-style coordination also indicate that a more complete information view can help coordination, but this result depends on the underlying network structure. One implication from this result is that when considering how we can improve collective performance it is worth considering the effect of network changes versus information views because they may both lead to the same outcome, but one may be less costly or have fewer possible negative effects.

\section{Discussion and conclusion}

In designing and building networks it is important to recognize that restricting the network can impede overall performance for a variety of reason and across a variety of tasks. Scholars have argued that adding connections to a network can reduce voter turnout, lead to greater traffic congestion, and make it more difficult for groups to organize a protest. The possibility that adding connections to a network degrades performance makes it crucial that we identify if there are other ways to modify the network setting that pose less risk of degrading performance. We demonstrate experimentally that giving individuals a more complete view of the network can improve coordination, without having to add connections that could impair the ability of a group to solve a coordination problem. Our experimental results show that for two different common coordination tasks, providing subjects with a more complete view of the network can improve coordination, but this effect depends on the structure of the network.

Although we have not directly examined the mechanisms by which a more complete view of the network improves coordination our experience with these experiments and many related network coordination experiments suggests a few possibilities. One possible mechanism is that by allowing subjects to observe more of the network and subjects' colors it is simply easier for them to identify the color that will lead to a consensus in those games. In both anticoordination and consensus a more complete view can also make it easier for nodes to identify the nodes in the network that may be relatively more important (i.e. those that are highly central), which can make it easier for actors to use the important node(s) as a focal point. In the anti-coordination task the ability to see more of the network and more subjects may help subjects observe the nodes that have not solved the coordination problem and then they may be able to change their color in a way that encourages others to also change their color and thereby resolve the conflict(s) in the network. It is important to point out that we have not directly tested these mechanisms in our experiments, and doing so would require the ability to directly manipulate both the treatment and the mechanism under study (Imai et al., 2011). Although we have not directly tested the mechanisms, the experimental findings occur across different tasks and network structures, which gives us increased confidence that these results would hold in other settings. These results have implications for both scholarly research and the design of networks.

In empirical research we may need to consider what subjects know about the overall network structure, because these results suggest that if individuals possess considerable knowledge of the entire network then different network structures may have different outcomes. To put it another way, to demonstrate that a network affects behavior we should account for both the structure of the network and also the information environment in which people make choices. If we fail to do this, then when we compare across network structures we may infer it is the structure that leads to different performance when in fact it may be the information view. More specifically, our results demonstrate that with significant information about network structure we may not observe differences across network environments even though such differences would exist in the absence of a complete view of the network. In both cases, the possible interaction between structure and information views makes it crucial to account for both in our empirical research. These results can help us to understand the conditions under which we may observe effects of network structure on behavior.

In situations where network design is a concern (i.e. designing institutions or building new connections in an existing network) these results suggest that it may be possible to gain the benefits of additional connections by providing individuals with a more complete view of the network. For example, a prominent former Amazon.com engineer has argued that one of Amazon's chief competitive advantages is that employees have essentially a complete view of the network (Yegge, 2011). Specifically, he recounts how Amazon CEO Jeff Bezos insisted that employees communicate via a universally available interface that allows every node (engineering team) to view the action of every other node, so that "there will be no [private] communication allowed: no direct linking, no direct reads of another team's data store, no shared-memory model, no back-doors whatsoever. The only communication allowed is via service interface calls over the [universally viewable] network." (Yegge, 2011)

Social network sites such as Facebook and their users have recognized that changing the view of the network can have benefits by making it possible for individuals to share content with their friends' friends, with entire subgraphs, or even publically. In social media, however, the information view also raises important individual privacy concerns even if there might be collective benefits to more complete information views.

In other settings (such as the design of networks for legislators to monitor agencies) it may be that adding direct connections between legislators and more and more agencies and sub-agencies actually makes the monitoring process more difficult. Alternatives to direct connections include legislators requiring agencies to report publicly on the actions of their sub-agencies (i.e. a more complete view of the agency) and the aforementioned "fire-alarm networks" in which legislators provide other actors with a more complete view of the network and the ability to alert legislators to important activity. This more complete view may facilitate a coordinated response by congressional actors without the impediments that more connections in the network might add.

Overall there is reason to believe that when designing networks it may be valuable to consider alternatives to changes in the structure of the network because changing the network may impede, rather than improve, performance. We have shown that when a group faces a coordination task it may improve outcomes to provide individuals with a more complete view of the network. This finding can help improve our research on network design and guide our construction or modification of existing networks.

\section{References}

Aberbach, J., 1990. Keeping a Watchful Eye: The Politics of Congressional Oversight. Brookings Press, Washington, DC.

Braess, D., 1969. Über ein Paradoxon aus der Verkehrsplanung. Unternehmensforschung 12, 258-268, Translation of the Braess 1968 article from German to English appears as the article "On a paradox of traffic planning," by D. Braess, A. Nagurney, and T. Wakolbinger in the Journal Transportation Science, volume 39, 2005, pp. 446-450.

Bramoullé, Y., Kranton, R., 2007. Public goods in networks. Journal of Economic Theory $135,478-494$

Bradley, C.A., Kelly, J., 2008. The concept of international delegation. Law and Contemporary Problems 71, 1.

Bradbury, E.M., Davidson, R.A., Lawrence Evans, C., 2008. The senate whip system an exploration. In: Monroe, N., Roberts, J., Rohde, D. (Eds.), Why Not Parties? Party Effects in the United States Senate. University of Chicago Press, Chicago, IL.

Calvert, R., 1992. Leadership and its basis in problems of social coordination. International Political Science Review 13 (1), 7-24. 
Cassar, A., 2007. Coordination and cooperation in local, random and small world networks: experimental evidence. Games and Economic Behavior 58, 209-230.

Choi, S., Gale, D., Kariv, S., Palfrey, T., 2008. Network architecture, salience, and coordination. Working Paper.

Chong, D., 1991. Collective Action and the Civil Rights Movement. University of Chicago Press, Chicago, IL.

Chung, F., Young, S., 2010. Braess's Paradox in Large Sparse Graphs. Internet and Network Economics, 194-208.

Chwe, M.S.Y., 2000. Communication and coordination in social networks. The Review of Economic Studies 67 (1), 1-16.

Cowhey, P., Mueller, M., 2009. Network Theory and Internet Governance. In: Kahler, M. (Ed.), Networked Politics. Cornell University Press.

Dow, J., Werlang, S.R.C., 1994. Nash equilibrium under Knightian uncertainty: breaking down backward induction. Journal of Economic Theory 64, 304-324.

Easley, D., Kleinberg, J., 2010. Networks, crowds, and markets. Cambridge University Press.

Ebel, H., Mielsch, L.I., Bornholdt, S., 2002. Scale-free topology of e-mail networks. Physical Review E 66.

Echenique, F., Yariv, L., 2011. An experimental study of decentralized matching. Working Paper.

Enemark, D., McCubbins, M., Paturi, R., Weller, N., 2011. Does more connectivity help groups to solve social problems? In: Proceedings of the 12th ACM Conference on Electronic Commerce.

Fowler, J.H., 2005. Turnout in a small world. In: Zuckerman, A. (Ed.), Social Logic of Politics. Temple University Press, Philadelphia, pp. 269-287.

Gleditsch, K.S., Ward, M.D., 2006. Diffusion and the international context of democratization. International Organization 60 (4), 911-933.

Gleditsch, K.S., Ward, M.D., 2008. Diffusion and the spread of democratic institutions. In: Dobbins, F., Garrett, G., Simmons, B. (Eds.), The Global Diffusion of Democracy and Markets. Cambridge University Press, Cambridge, pp. 261-302.

Gould, R.V., 1993. Collective action and network structure. American Sociological Review 58 (2), 182-196.

Grannovetter, M., 1974. Getting a Job: A Study of Contacts and Careers. Harvard University, Cambridge, MA

Hafner-Burton, E., Montgomery, A.H., 2006. Power positions: international organizations, social networks, and conflict. Journal of Conflict Resolution 50 (1), $3-27$.

Hafner-Burton, E., Montgomery, A.H., 2008. Power or plenty: how do international trade institutions affect economic sanctions? Journal of Conflict Resolution 52 (April (2)), 213-242.

Hafner-Burton, E., Kahler, M., Montgomery, A.H., 2009. Network analysis for international relations. International Organization 63 (3), 559-592.

Imai, Kosuke, Keele, L., Tingley, D., Yamamoto, T., 2011. Unpacking the black box of causality: learning about causal mechanisms from experimental and observational Studies. American Political Science Review 105 (4), 765-789.

Jackson, M., Rodriguez-Barraquer, T., Tan, X., 2012. Social capital and social quilts: network patterns of favor exchange. American Economic Review 102 (5), 1857-1897.

Kahler, M., 2009. Networked politics: agency, power, and governance. In: Kahler, M. (Ed.), Networked Politics: Agency, Power, and Governance. Cornell University Press, Ithaca, NY, pp. 1-20.

Kearns, M., Suri, S., Montfort, N., 2006. An experimental study of the coloring problem on human subject networks. Science 313, 824 .

Knight, F., 1921. Risk, Uncertainty and Profit. Houghton Mifflin, Boston.

Kolata, G., 1990. What if they closed 42nd street and nobody noticed? New York Times December, 38.

King, G., Zeng, L., 2006. The dangers of extreme counterfactuals. Political Analysis 14, 131-159.
Lazer, D., Friedman, A., 2007. The network structure of exploration and exploitation. Administrative Science Quarterly 52 (4), 667-694.

Leskovec, J., Lang, K.J., Dasgupta, A., Mahoney, M., 2009. Community structure in large networks: natural cluster sizes and the absence of large well-defined clusters. Internet Mathematics 6 (1), 29-123.

Lewis, D., 1969. Convention: A Philosophical Study. Harvard University Press, Cambridge, MA.

Mackie, G., 1996. Ending footbinding and infibulation: a convention account. American Sociological Review 61, 999-1017.

Maoz, Z., 2006. Network polarization, network interdependence, and international conflict, 1816-2002. Journal of Peace Research 43 (4), 391-411.

Maoz, Z., Terris, L.G., Kuperman, R.D., Talmud, I., 2007. Network centrality and international conflict: does it pay to be important? In: Friemel, T.N.(Ed.), Applications of Social Networks Analysis. Universitat Verlag Konstanz, Konstanz (Germany), pp. 121-151.

McClurg, S., 2003. Social networks and political participation: the role of social interaction in explaining political participation. Political Research Quarterly 56, 448-464.

McCubbins, M.D., Schwartz, T., 1984. Congressional oversight overlooked: police patrols versus fire alarms. American Journal of Political Science 28, 16-79.

McCubbins, M., Paturi, R., Weller, N., 2009. Connected coordination: network structure and group coordination. American Politics Research September.

Morrow, J.D., 1994. Modeling the forms of international cooperation: distribution versus information. International Organization 48, 387.

Niou, E.M.S., Ordeshook, P.C., 1994. 'Less filling, tastes great': the realist-neoliberal debate. World Politics 46 (2), 209-234.

Rubin, D.B., 1990. Formal modes of statistical inference for causal effects. Journal of Statistical Planning and Inference 25, 279-292.

Schelling, T.C., 1960. The Strategy of Conflict. Harvard University Press, Cambridge.

Scholz, J., Berardo, R., Kile, B., 2008. Do networks solve collective action problems? Credibility search, and collaboration. Journal of Politics 70 (2), 393406.

Shadish, W., Cook, T.D., Campbell, D.T., 2001. Experimental and Quasi-Experimental Designs for Generalized Causal Inference. Houghton Mifflin Company, New York.

Siegel, David A., 2009. Social networks and collective action. American Journal of Political Science 53 (1), 122-138.

Simmel, G., 1950. The Sociology of Georg Simmel Kurt Wolff tran. Free Press, New York.

Simmons, B.A., Elkins, Z., 2005. On waves, clusters and diffusion: a conceptual framework. Annals of the American Academy of Political and Social Science 598, 33-51.

Snidal, D., 1985. Coordination versus prisoners' dilemma: implications for international cooperation and regimes. American Political Science Review 79 (4), 923-942.

Stein, A., 1982. Coordination and collaboration: regimes in an anarchic world. International Organization 36 (2), 299-324, International Regimes (Spring, 1982).

Suri, S., Watts, D.J., 2011. Cooperation and contagion in web-based, networked public goods experiments. PLoS ONE 6 (3), e16836, http://dx.doi.org/10.1371/journal.pone.001683.

Ward, M.D., Stovel, K., Sacks, A., 2011. Network analysis and political science. In: Levi, M. (Ed.), Annual Review of Political Science, vol. 14, pp. 245-246.

Watts, D., 2003. Six Degrees: The Science of a Connected Age. Norton, New York.

Watts, D.J., Strogatz, S.H., 1998. Collective dynamics of 'small-world' networks. Nature 393 (6684), 409-410.

Weingast, B.R., 1997. The political foundations of democracy and the rule of law. American Political Science Review 91 (June), 245-263.

Wilson, R.K., Rhodes, C.M., 1997. Leadership and credibility in n-person coordination games. Journal of Conflict Resolution 41, 767-791.

Yegge, S., 2011. Google Platforms Rant, Retrieved October 23, 2012 from https://plus.google.com/112678702228711889851/posts/eVeouesvaVX 